University of Nebraska - Lincoln

DigitalCommons@University of Nebraska - Lincoln

Faculty Publications from Nebraska Center for

Materials and Nanoscience, Nebraska Center

Materials and Nanoscience

for (NCMN)

2009

\title{
Optical, structural, and magnetic properties of cobalt nanostructure thin films
}

D. Schmidt

University of Nebraska-Lincoln

A. C. Kjerstad

University of Nebraska-Lincoln

Tino Hofmann

University of Nebraska - Lincoln, thofmann4@unl.edu

Ralph Skomski

University of Nebraska at Lincoln, rskomski2@unl.edu

Eva Schubert

University of Nebraska - Lincoln, efranke3@unl.edu

See next page for additional authors

Follow this and additional works at: https://digitalcommons.unl.edu/cmrafacpub

Part of the Nanoscience and Nanotechnology Commons

Schmidt, D.; Kjerstad, A. C.; Hofmann, Tino; Skomski, Ralph; Schubert, Eva; and Schubert, Mathias, "Optical, structural, and magnetic properties of cobalt nanostructure thin films" (2009). Faculty Publications from Nebraska Center for Materials and Nanoscience. 100.

https://digitalcommons.unl.edu/cmrafacpub/100

This Article is brought to you for free and open access by the Materials and Nanoscience, Nebraska Center for (NCMN) at DigitalCommons@University of Nebraska - Lincoln. It has been accepted for inclusion in Faculty Publications from Nebraska Center for Materials and Nanoscience by an authorized administrator of DigitalCommons@University of Nebraska - Lincoln. 


\section{Authors}

D. Schmidt, A. C. Kjerstad, Tino Hofmann, Ralph Skomski, Eva Schubert, and Mathias Schubert 


\title{
Optical, structural, and magnetic properties of cobalt nanostructure thin films
}

\author{
D. Schmidt, ${ }^{1, a)}$ A. C. Kjerstad, ${ }^{1}$ T. Hofmann, ${ }^{1}$ R. Skomski, ${ }^{2}$ E. Schubert, ${ }^{1}$ and \\ M. Schubert ${ }^{1}$ \\ ${ }^{1}$ Department of Electrical Engineering and Nebraska Center for Materials and Nanoscience, \\ University of Nebraska-Lincoln, Lincoln, Nebraska 68588-0511, USA \\ ${ }^{2}$ Department of Physics and Astronomy and Nebraska Center for Materials and Nanoscience, \\ University of Nebraska-Lincoln, Lincoln, Nebraska 68588-0111, USA
}

(Received 13 March 2009; accepted 23 April 2009; published online 2 June 2009)

\begin{abstract}
We report on optical, structural, and magnetic properties of two substantially different cobalt nanostructure thin films deposited at an oblique angle of incidence of $85^{\circ}$ away from the substrate normal. Comparison is made between an achiral columnar thin film grown without substrate rotation and a chiral nanocoil sculptured thin film by glancing angle deposition with substrate rotation. Generalized spectroscopic ellipsometry is employed to determine geometrical structure properties and the anisotropic optical constants of the films in the spectral range from 400 to $1000 \mathrm{~nm}$. The magnetic properties are analyzed with a superconducting quantum interference device magnetometer. Both nanostructure thin films show highly anisotropic optical properties such as strong form birefringence and large dichroism. In particular, Co slanted columnar thin films are found to possess monoclinic optical properties. Magnetic measurements at room temperature show hysteresis anisotropy with respect to a magnetic field either parallel or perpendicular to the nanostructures' long axis. We find extremely large coercive fields of approximately $3 \mathrm{kOe}$ for our achiral columnar nanostructures. (C) 2009 American Institute of Physics. [DOI: 10.1063/1.3138809]
\end{abstract}

\section{INTRODUCTION}

Advanced growth techniques allow for engineering metamaterials composed of three-dimensional highly anisotropic structures on the nanoscale. ${ }^{1}$ This has opened the new realm of tailoring desired physical properties along specific directions in space. Nanostructured materials have been already employed for several interesting sensor applications ${ }^{2,3}$ and functional optical coatings, ${ }^{4,5}$ for example. However, intrinsic physical properties are not fully understood yet. In general, mechanical, electrical, optical, or magnetic properties of structured thin films in the nanometer range differ drastically from the bulk material due to their small size or shape anisotropy. ${ }^{6,7}$ Consequently, form-induced polarization confinement and quantization effects need to be taken into account. It is crucial to understand intrinsic physical properties such as polarizabilities or magnetic hysteresis anisotropy of such three-dimensionally sculptured thin films to tailor new artificial materials for exploring future applications. In particular, ferromagnetic materials such as cobalt are interesting since magnetic and optical properties may be tailored at the same time. Hence, in a chiral ferromagnetic material on the nanoscale, cross-coupling effects between optical activity and magnetic-field induced changes in the optical properties may be observable similar to what has been discovered in molecules earlier. ${ }^{8}$

Glancing angle deposition (GLAD) allows for engineering of sculptured thin films nanostructures. ${ }^{1}$ This technique utilizes the characteristics of an impinging particle flux at very oblique angle of incidence (typically $>80^{\circ}$ ) onto a sub-

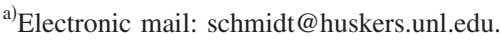

strate. A concurrent growth mechanism due to geometrical self-shadowing in combination with limited adatom mobility governs the deposition process. Paired with a controlled substrate motion GLAD allows for a self-organized bottom-up fabrication of three-dimensional nanostructures. Consequently, without prepatterning the substrate, randomly distributed but highly orientationally coherent discrete structures ranging from slanted or straight columns to screws and helices with sizes from a few tens of nanometers up to micrometers can be deposited. Furthermore, a broad range of materials such as semiconductors, dielectrics, or metals can be tailored with this technique. ${ }^{9}$

Magnetic properties on GLAD growth of randomly and periodically arranged ferromagnetic nanostructures have been published with strong emphasis on magnetic hysteresis investigations. Liu et al. ${ }^{10}$ found that the magnetic anisotropy and squareness ratio strongly depend on the angle of incidence of the vapor flux. Lisfi and Lodder ${ }^{11}$ found that there is also a strong dependence between growth angle and domain structure in obliquely sputtered Co thin films. Dick et al. ${ }^{12}$ reported on in-plane and out-of-plane coercivities of randomly and periodically arranged Co posts. However, direct comparison seems difficult because of different morphologies. Recently, Tang et al. ${ }^{13}$ found that the coercivity $H_{c}$ is constant along the direction perpendicular to the incident vapor beam whereas $H_{c}$ measured parallel to direction of vapor flux increases for particle flux incidence angles greater than $60^{\circ}$.

The optical properties of nanostructured surfaces have been studied by several groups and different approaches. Mbise et al. ${ }^{14}$ reported extensively about angular selective transmittance of slanted columnar thin films from different 
materials with a potential application for window coatings. Rovira et al. ${ }^{15}$ investigated the phenomenon of optical activity and determined optical rotation of helical structured thin films, and Jensen and Brett ${ }^{16}$ reported on reflectance and transmittance of three-dimensional photonic band gap crystals composed of square spirals, for example. Zhang and Zhao ${ }^{17}$ theoretically investigated the pitch-height dependent optical extinction spectra of helical Ag nanostructures by the discrete dipole approximation. Approaches were done to model experimental data of straight $\mathrm{GaSb}$ nanocones, ${ }^{18}$ straight Si nanorods, ${ }^{19,20}$ and slanted Si nanocolumns ${ }^{21}$ with anisotropic or graded effective medium approximations in order to extract structural properties such as film thickness and porosity, for instance. Among these, only Kaminska et $a l .{ }^{19}$ reported on optical constants in the visible spectral region of their uniaxial straight $\mathrm{Si}$ posts with nominal thicknesses ranging from 70 to $1000 \mathrm{~nm}$. However, effective medium approximations are valid only if the structure size is negligible against the wavelength, and effective medium considerations were consequently not applied in the present study. ${ }^{22}$

Nevertheless, nondestructive optical techniques such as ellipsometry have proven to be extremely suitable for determining structural (major polarization axes' orientation) and physical (dielectric tensor) properties of highly anisotropic thin films. It has been recently reported for orthorhombic ${ }^{23}$ and triclinic ${ }^{24}$ thin films as well as for monoclinic slanted columnar thin films from $\mathrm{Ti}$ and $\mathrm{Cr}^{25,26}$

In this paper we study the structural, optical, and magnetic properties of GLAD grown highly orientationally coherent Co nanostructures by generalized ellipsometry (GE) and superconducting quantum interference device (SQUID) magnetometer investigations. We demonstrate that GE analysis is not only limited to accurately determine principal optical constants and intrinsic birefringence but also has excellent capabilities in identifying geometrical parameters such as orientation and inclination of slanted nanostructures and additionally the pitch (number of turns per unit length) thickness of helical sculptured thin films.

\section{THEORY}

GE allows for determination of complete and accurate sets of optical constants for biaxial (dielectrically anisotropic) materials. ${ }^{27,28}$ For arbitrarily anisotropic materials, the complex ratio $\rho$ of the $s$ - and $p$-polarized reflectivities depends on the polarization state of the incident light. Measurement of $\rho$ can be addressed within different presentations of the electromagnetic plane wave response. Here we make use of the Stokes descriptive system where real-valued Mueller matrix elements $M_{i j}$ connect the Stokes parameters before and after sample interaction. ${ }^{29}$ The Stokes vector elements for the traditional $p$ - $s$ polarization system are $S_{0}=I_{p}+I_{s}, S_{1}$ $=I_{p}-I_{s}, S_{2}=I_{45}-I_{-45}$, and $S_{3}=I_{\sigma+}-I_{\sigma-}$, where $I_{p}, I_{s}, I_{45}, I_{-45}$, $I_{\sigma+}$, and $I_{\sigma_{-}}$denote the intensities for the $p-, s_{-},+45^{\circ},-45^{\circ}$, right-, and left-handed circularly polarized light components, respectively. Data analysis requires nonlinear regression methods, where measured and calculated GE data are matched as close as possible by varying appropriate physical model parameters. Due to the complexity of this subject, thorough discussion of this issue is beyond the scope of this paper, and referral is made to literature. ${ }^{23,24,28-33}$ The linear polarizability response of structured thin films due to an electric field $\mathbf{E}$ is a superposition of contributions along certain directions $\mathbf{a}=a_{x} \mathbf{x}+a_{y} \mathbf{y}+a_{z} \mathbf{z}, \quad \mathbf{b}=b_{x} \mathbf{x}+b_{y} \mathbf{y}+b_{z} \mathbf{z}$, and $\mathbf{c}=c_{x} \mathbf{x}$ $+c_{y} \mathbf{y}+c_{z} \mathbf{Z}: \mathbf{P}=\varrho_{a} \mathbf{a}+\varrho_{\mathbf{b}} \mathbf{b}+\varrho_{c} \mathbf{c}$ that are phenomenologically equivalent to (but physically different from) the crystallographic unit-cell vectors in bulk materials. In the laboratory Cartesian coordinate system the structured thin film is described by the second rank polarizability tensor $\chi$ as follows:

$$
\mathbf{P}=(1-\varepsilon) \mathbf{E}=\chi \mathbf{E}=\left(\begin{array}{ccc}
\chi_{x x} & \chi_{x y} & \chi_{x z} \\
\chi_{y x} & \chi_{y y} & \chi_{y z} \\
\chi_{z x} & \chi_{z y} & \chi_{z z}
\end{array}\right) \mathbf{E} .
$$

The Cartesian coordinate system $(x, y, z)$ is defined by the plane of incidence $(x, z)$ and the sample surface $(x, y)$. This Cartesian frame is rotated by the Euler angles $\phi, \psi$, and $\theta$ to an auxiliary system $(\xi, \eta, \zeta)$ with $\zeta$ being parallel to c. ${ }^{29,32}$ For orthorhombic, tetragonal, hexagonal, and trigonal systems $\phi, \psi$, and $\theta$ exist with $\chi$ being diagonal in $(\xi, \eta, \zeta)$. For monoclinic and triclinic systems an additional projection operation $\mathbf{T}$ onto the orthogonal auxiliary system $(\xi, \eta, \zeta)$ is necessary, ${ }^{34}$

$$
T=\left(\begin{array}{ccc}
\sin \beta & \frac{\cos \gamma-\cos \beta \cos \alpha}{\sin \beta} & 0 \\
0 & \sqrt{\sin ^{2} \alpha-\frac{\cos \gamma-\cos \beta \cos \alpha}{\sin \beta}} & 0 \\
\cos \beta & \cos \alpha & 1
\end{array}\right) .
$$

Additional parameters $\alpha, \beta$, and $\gamma$ are introduced into the analysis procedure, which differentiate between orthorhombic $\left(\alpha=\beta=\gamma=90^{\circ}\right)$, monoclinic $\left(\beta \neq 90^{\circ}\right)$, or triclinic $(\alpha \neq \beta \neq \gamma)$ biaxial optical properties. As a result, one obtains major-axis polarization response functions $\varrho_{a}, \varrho_{b}$, and $\varrho_{c}$, Euler angles $\phi, \psi$, and $\theta$; internal angles $\alpha, \beta$, and $\gamma$; and thin film thickness $d$. Note that angle and thickness parameters are not allowed to vary with wavelength. With respect to helical thin films a homogenous rotation is introduced to such an arbitrarily oriented coordinate system. A detailed discussion can be found elsewhere. ${ }^{30,35}$ The major optical constants $\left(n_{j}+\sqrt{-1} k_{j}\right) \equiv \sqrt{\left(1+\varrho_{j}\right)}(j=a, b, c)$ can thus be extracted on a point-by-point basis, i.e., without any physical lineshape implementations. Kramers-Kronig consistency tests can then be done individually for dielectric functions along each axis. ${ }^{24}$

\section{EXPERIMENTAL}

Cobalt nanostructures were deposited at room temperature by electron-beam GLAD in a customized ultrahigh vacuum chamber onto silicon substrates. The (001) $p$-type $\mathrm{Si}$ substrates had a native oxide layer of approximately $3 \mathrm{~nm}$. The distance between source and the (xyz) sample manipulator is $460 \mathrm{~mm}$. The deposition angle measured between the incident particle flux direction and the substrate normal was set to $85^{\circ}$. A bulklike Co thin film with thickness of approximately $600 \mathrm{~nm}$ was prepared at normal incidence for purpose 


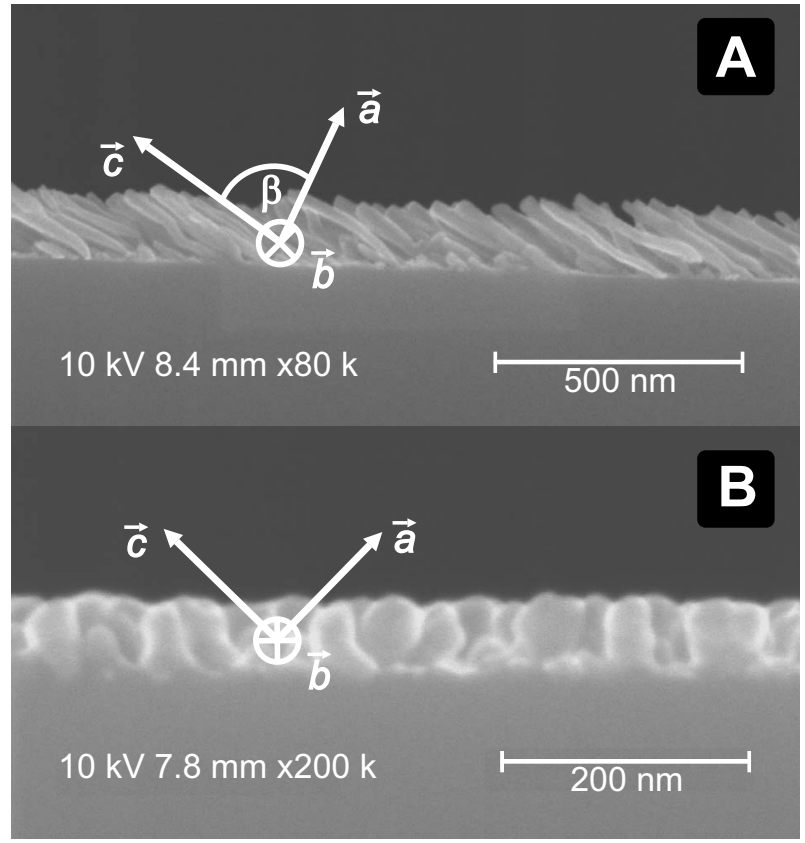

FIG. 1. SEM micrograph of slanted Co nanocolumns (a) and Co nanocoils (b). The overlaid coordinate system in (a) indicates the orientation of the fixed biaxial system with its internal c-axis along the nanocolumns and b-axis parallel to the film interface. The monoclinic angle $\beta$ between axes a and $\mathbf{c}$ is $80.6^{\circ}$. In (b) the system is orthorhombic with axes a and $\mathbf{c}$ inclined toward the sample normal. The orthorhombic system rotates around the sample normal along the chiral nanowires (z-direction, Fig. 2). The b-axis is tilted by $2.2^{\circ}$ with respect to the substrate surface.

of comparison. Figure 1 depicts a high-resolution fieldemission scanning electron microscope (SEM) image of an edge view of both samples under investigation. The achiral slanted columnar thin film [Fig. 1(a)] was deposited without substrate rotation whereas a counterclockwise substrate rotation at $0.25 \mathrm{rpm}$ results in growth of the chiral sculptured thin film (nanocoils) shown in Fig. 1(b). Angle-resolved (angle-of-incidence $\Phi_{A}$ and in-plane rotation angle $\varphi$ ) spectroscopic Mueller matrix ellipsometry measurements were performed using a commercial instrument $\left(\mathrm{M} 2000^{\mathrm{TM}}\right.$, J. A. Woollam Co., Inc.) within the spectral range from 400 to $1000 \mathrm{~nm}$. The ellipsometer was mounted on an automatic variable $\Phi_{A}$ and sample rotator $\varphi$ stage. $\Phi_{A}$ was varied from $45^{\circ}$ to $75^{\circ}$ in steps of $10^{\circ}$, while $\varphi$ was varied from $0^{\circ}$ to $360^{\circ}$ in steps of $5^{\circ}$. The polarizer-compensator-sampleanalyzer ellipsometer is capable of measuring 11 out of 16 Mueller matrix elements normalized to $M_{11}$ (except for elements in fourth row). ${ }^{29}$ The $\mathrm{x}$-ray diffraction (XRD) measurements were carried out with a Rigaku $\mathrm{X}$-ray diffractometer (Multiflex+), which operates with $\mathrm{Cu} K \alpha$ radiation. Magnetization properties (hysteresis loops) were recorded with a SQUID (MPMS XL7, Quantum Design). Room temperature measurements were done with the magnetic-field direction aligned either parallel or perpendicular to the long axis of the nanostructures, i.e., parallel and perpendicular to the slanted nanocolumns, and parallel and perpendicular to the substrate normal for the chiral nanocoils. Measurements were taken up to $12 \mathrm{kOe}$.

The experimentally obtained Mueller matrix spectra for the achiral slanted columnar thin film were modeled using a

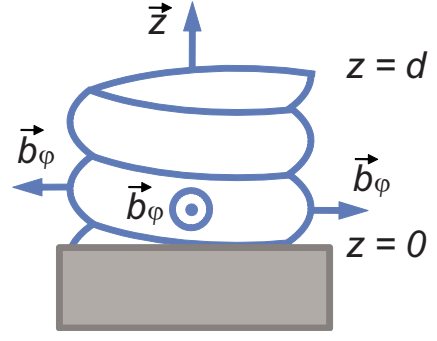

FIG. 2. (Color online) Schematic representation of a single chiral nanocoil shown in Fig. 1(b). The in-plane orientation $\varphi$ of the intrinsic coordinate system, which rotates stepwise for each from the substrate interface $(z=0)$ to the top of the structures $(z=d)$, is denoted by different orientations of the b-axis $\left(\mathbf{b}_{\varphi}\right)$.

single anisotropic layer on top of a $\mathrm{Si}$ substrate. Consequently, the computed Euler angles $(\phi, \theta$, and $\psi)$ that transform the Cartesian coordinate system $(x, y, z)$ into the sample coordinates $(\xi, \eta, \zeta)$ represent the orientation of the nanostructure and the internal angles $\alpha, \beta$, and $\gamma$ define the biaxial properties of the film. Furthermore, the overall thickness and the dielectric tensor, with three major elements $\varepsilon_{a}, \varepsilon_{b}$, and $\varepsilon_{c}$, pertinent to the intrinsic axes $\mathbf{a}, \mathbf{b}$, and $\mathbf{c}$, are model parameters.

The nanocoils were described by a graded biaxial (orthorhombic) absorbing layer, which was virtually split up in five slices with dielectric function tensor rotated stepwise with respect to the adjacent layer according to the handedness. This homogenous in-plane rotation $\varphi$ from the substrate interface at $z=0$ to the top of the structure at $z=d$ accounts for the helical structure of the chiral nanocoils, which was introduced during the growth process by rotating the substrate with respect to the incoming particle flux (Fig. 2). Such chiral sculptured thin films can be considered as "frozen" cholesteric liquid crystals. ${ }^{27}$ The subdivision of the chiral nanostructure into five homogeneous anisotropic layers with piecewise rotated orthorhombic axis system $(\mathbf{a}, \mathbf{b}, \mathbf{c} ; \alpha=\beta$ $=\gamma=90^{\circ}$ ) was sufficient to reach close match between model and experiment. While the subdivision into five sublayers only is a simple approximation, the increase in sublayers thereby refining the homogeneous rotation approximation resulted in no further best-match model improvement. We explain this finding by the very small features of the nanocolumns. The Euler angles $\theta$ and $\psi$ and an offset angle $\varphi_{0}$ along with the overall thickness complete the list of model parameters necessary to describe the optical properties of this chiral sculptured thin film. Best-match model analysis of wavelength-dependent GE data measured at multiple angleof-incidence $\Phi_{a}$, and multiple sample in-pane-azimuth orientations $\varphi$ can potentially provide all these parameters nondestructively.

\section{RESULTS AND DISCUSSION}

\section{A. Optical and structural properties}

Figure 3 depicts selected Mueller matrix data (GE) obtained from both Co nanostructures, shown in Fig. 1, at an exemplary wavelength of $\lambda=850 \mathrm{~nm}$. Mueller matrix elements not shown in the figure can be obtained by symmetry as described earlier. ${ }^{33}$ Note that model and experimental data 

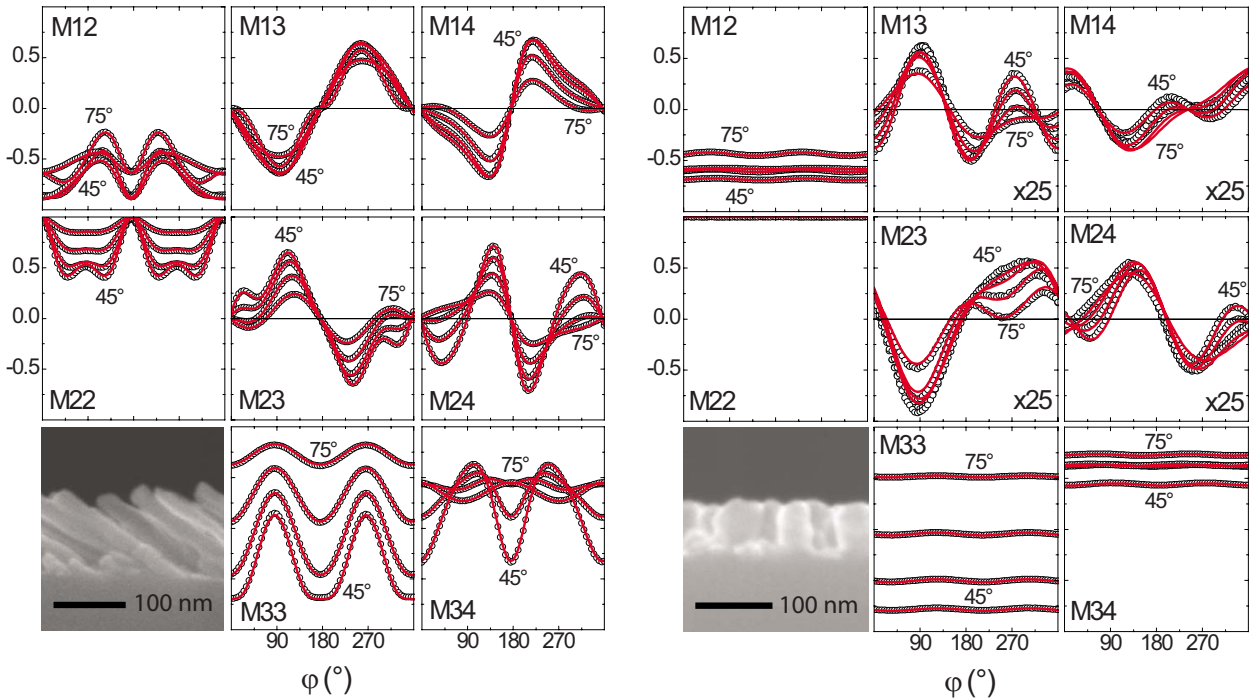

FIG. 3. (Color online) Exemplary experimental (circles) and best-match calculated (solid lines) GE data of slanted columnar thin film (left) and nanocoils (right) vs sample azimuth angle $\varphi$ and angle-of-incidence $\Phi_{A}$ at $\lambda=850 \mathrm{~nm}$. Note the excellent match between model and experiment. The numbers in the right panel correspond to the magnification of the data shown. Note that the chiral nanostructures do not reveal an isotropy orientation; i.e., a sample position $\varphi$ at which all offdiagonal Mueller matrix data $\left(M_{13}, M_{14}, M_{23}, M_{24}\right) \quad$ vanish, but which is the case near $\varphi=0^{\circ}$ and $\varphi$ $=180^{\circ}$ for the nanocolumns. are in perfect agreement in both cases for all wavelengths in the investigated spectral region from 400 to $1000 \mathrm{~nm}$ (data omitted for brevity here). The off-diagonal Mueller matrix data $\left(M_{13}, M_{14}, M_{23}, M_{24}\right)$ exhibit the highly anisotropic nature of the Co nanostructures. These elements are zero for all angles of incidences $\Phi_{A}$ at all wavelengths for isotropic samples. For the slanted columnar Co thin film pseudoisotropic sample orientations can be identified at $\varphi \approx 0^{\circ}$ and $\varphi$ $\approx 180^{\circ}$, which coincide with orientations of the sample when the tilting direction of the nanocolumns is parallel to the plane of incidence. Hence, no $p$-polarized light is converted into $s$-polarized light and vice versa in this particular setup. We further obtained that the slanted Co columnar thin film possesses monoclinic optical properties with an angle $\beta$ $=83.9^{\circ}$, and which is in agreement with our previous reports on similar thin films from $\mathrm{Ti}$ and $\mathrm{Cr}^{25,26}$ The intrinsic monoclinic coordinate system, which is fixed in space, is depicted in Fig. 1(a). This monoclinic angle can be understood as a structural property of slanted columnar thin films. At the bottom of the structure, charge exchange is possible due to a conducting nucleation layer whereas this is not possible at the isolated top of the columns. Therefore, the overall dipole moment for electric fields perpendicular to the columns and within the slanting plane is tilted toward the surface normal.

In contrast, our model calculations for the chiral nanocoils reveal that the dielectric function tensor pertinent to an orthorhombic coordinate system is not fixed in space but rather rotating with the c-axis along the windings. Here, the assumed screening of next neighbors, which may be responsible for the monoclinic properties in case of the slanted nanocolumns, is not an issue since the structures are parallel to the surface normal. The modeled amount of overall turns $\varphi$ from $z=0$ to $z=d$ (see Fig. 2) is in perfect agreement with the actual amount of substrate rotations during the growth process. This rotation of the intrinsic axes $(\mathbf{a}, \mathbf{b}$, and $\mathbf{c})$ is responsible for the absence of pseudoisotropic sample orientations, which are, in contrast, present for the slanted columnar thin film with a fixed intrinsic coordinate system. Hence, even though the elongation of the nanocoils is strongly reduced with no separation between individual turns the optical properties have nothing in common with a uniaxial ma- terial as, for example, a layer of straight posts parallel to the surface normal. In such a structured thin film the coordinate system is oriented with the c-axis parallel to the surface normal (along the long axis of the nanostructure). ${ }^{6}$ This gives rise to the assumption that the internal structure of the nanocoils is different from that of the slanted columns.

Table I summarizes the optical and structural properties of both investigated samples and reveals that SEM and GE values are in excellent agreement. Both methods result in a film thickness for the nanocoil sample of around $63 \mathrm{~nm}$ and an inclination angle for the slanted columnar thin film of approximately $\theta=55.5^{\circ}$, for instance. Note that inclination angles are measured with respect to the surface normal. In case of the nanocoil sample this inclination angle expresses the tilt of the c-axis, which is considered to describe the outer radius of the coil and thus determines the slope of the coil windings. Furthermore, we found that the b-axis is tilted by $2.2^{\circ}$ with respect to the substrate surface. Knowing the total film thickness $d$, the c-axis inclination angle $\theta$, and the overall in-plane rotation $\varphi$ the pitch $P$ and consequently the diameter $d_{\text {coil }}$ of a single coil can be computed as $d_{\text {coil }}$ $=P / \tan \theta$. This diameter is with $43.6 \mathrm{~nm}$ as well in excellent agreement with the estimate of $40 \mathrm{~nm}$ according to the SEM micrograph and thus almost only twice the diameter of a single slanted Co column. The a- and b-axis orientations with respect to the substrate surface normal can be understood as effective polarization radii of the chiral nanostructures, and result in approximately 37 and $739 \mathrm{~nm}$, respectively. One may interpret these radii as effective coupling distances within which the individual nanostructures couple

TABLE I. Summary of thickness $d$, c-axis inclination angle $\theta$, and structure diameter $d_{\varnothing}$ of our nanostructured Co thin films.

\begin{tabular}{lccccc}
\hline \hline & \multicolumn{2}{c}{ Slanted columns } & & \multicolumn{2}{c}{ Nanocoils } \\
\cline { 2 - 3 } \cline { 5 - 6 } & SEM & GE & & SEM & GE \\
\hline$d$ & $125 \mathrm{~nm}$ & $113.6 \mathrm{~nm}$ & & $63 \mathrm{~nm}$ & $63.7 \mathrm{~nm}$ \\
$\theta$ & $55.5^{\circ}$ & $55.3^{\circ}$ & & $\mathrm{a}$ & $37.5^{\circ}$ \\
$d_{\varnothing}$ & $25 \mathrm{~nm}$ & $\mathrm{a}$ & & $40 \mathrm{~nm}$ & $43.6 \mathrm{~nm}$ \\
\hline \hline
\end{tabular}

${ }^{\mathrm{a}}$ These values are not determinable with the respective technique. 


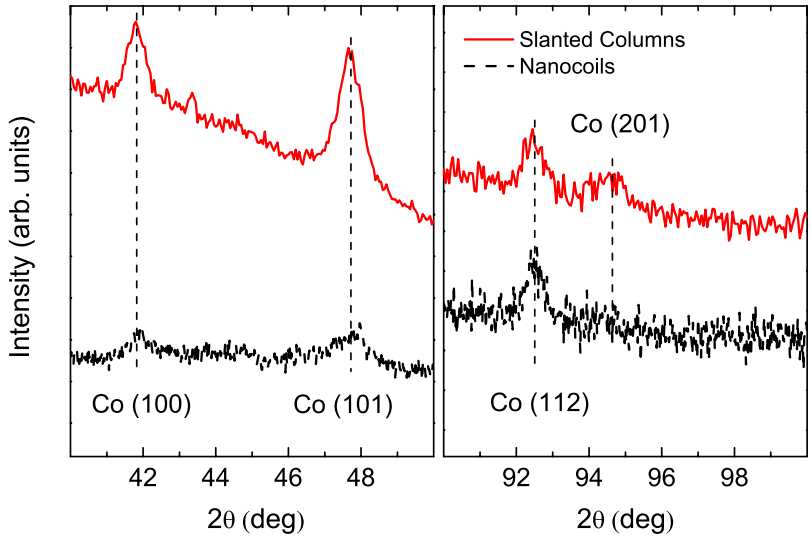

FIG. 4. (Color online) XRD spectra of slanted Co nanocolumns (solid) and Co nanocoils (broken) indicate that both Co structured thin films possess a hcp lattice structure.

their dielectric polarization response. The resultant aspect ratios are approximately 7 and 1.5 for the slanted nanocolumns and nanocoils, respectively. Note that the aspect ratio was calculated using the actual length of the nanocolumns of about $170 \mathrm{~nm}$.

XRD measurements suggest that the slanted columns have a largely monocrystalline, hexagonal close-packed (hcp) structure with c-axis parallel to the wires, which is in agreement with other reports of posts and nanowires made by GLAD and electrodeposition, respectively. ${ }^{12,36,37}$ Figure 4 reveals (100) peaks in both nanostructured films, which may originate from the nucleation layer. With respect to the slanted columnar thin film sample the other four peaks belonging to a Co hcp lattice may be due to a fraction of nanocolumns, which is tilted or bend slightly more or slightly less than the average $55^{\circ}$ determined by GE and SEM analysis. Tilting the c-axis only $3^{\circ}$ and $4^{\circ}$ away from $55^{\circ}$ exposes the (101) and (112) planes, respectively, considering a nondistorted Co hcp lattice. The fact that the same peaks with less intensity [except for (112) lattice planes] are observed for the nanocoil sample hints for a more homogeneous alignment and leads together with the optical analysis to the assumption that the nanocoils have the same crystallographic arrangement except for an additional in-plane rotation introduced during growth process.

Figure 5 depicts wavelength dependencies of the major axes' refractive indices $n_{i}$ and extinction coefficients $k_{i}$ (indices $i=a, b, c$ pertinent to axes of the coordinate system shown in Fig. 1) that differ drastically from those of Co bulk material. ${ }^{38}$ The obtained optical constant spectra sets were tested individually for all major polarization axes using model lineshape approaches confirming their KramersKronig consistency. The results of the model lineshape analysis and discussion of the underlying electronic polarizability mechanisms within the nanostructures will be presented elsewhere. Strong birefringence and dichroism can be observed in the investigated spectral region between all polarizabilities. The index of refraction $n_{c}$ along the slanted nanocolumns' c-axis crosses both other refractive indices $n_{b}$ and $n_{a}$ (left panel). This observation violates the general order of principal refractive indices for similar biaxial mediums $\left(n_{c}>n_{b}>n_{a}\right)$ reported earlier by Hodgkinson and $\mathrm{Wu},{ }^{6}$ and only is in agreement for wavelength greater than $\lambda$ $=670 \mathrm{~nm}$. Such intersections are not present in case of the nanocoils with respect to $n_{i}$ in the investigated spectral region. However, here the extinction coefficients $k_{a}$ and $k_{b}$ are cross at around $\lambda=800 \mathrm{~nm}$. In general, $n_{c}$ and $k_{c}$ of the slanted nanocolumns have a strong wavelength dependence in contrast to the optical constants along the a- and $\mathbf{b}$-axis. Note that there is almost no absorption parallel to the film interface. Considering the nanocoils, refractive indices $n_{i}$ and extinction coefficients $k_{i}$ have moderate wavelength dispersion along all axes. It can be stated that within the investigated spectral region both parameters $n_{c}$ and $k_{c}$ are largest. In general, the optical constants of both nanostructures are significantly different even though they are from the same material and dimensions are in the same order of magnitude.

\section{B. Magnetic properties}

Magnetic hysteresis loops where carried out at room temperature using a SQUID magnetometer and are shown in Fig. 6 normalized to maximum saturation magnetization $M_{s}^{*}$ reached within our experiment. In both cases the external $\mathbf{B}$ field was applied parallel and perpendicular to the long axis of the nanostructures. In comparison with our e-beam evaporated bulklike sample with a thickness of approximately 600 $\mathrm{nm}$ and evaporated at normal incidence, which exhibits a coercive field $H_{c} \approx 30$ Oe, both $\mathrm{Co}$ nanostructures show greatly enlarged coercivities. Interestingly, the coercive fields
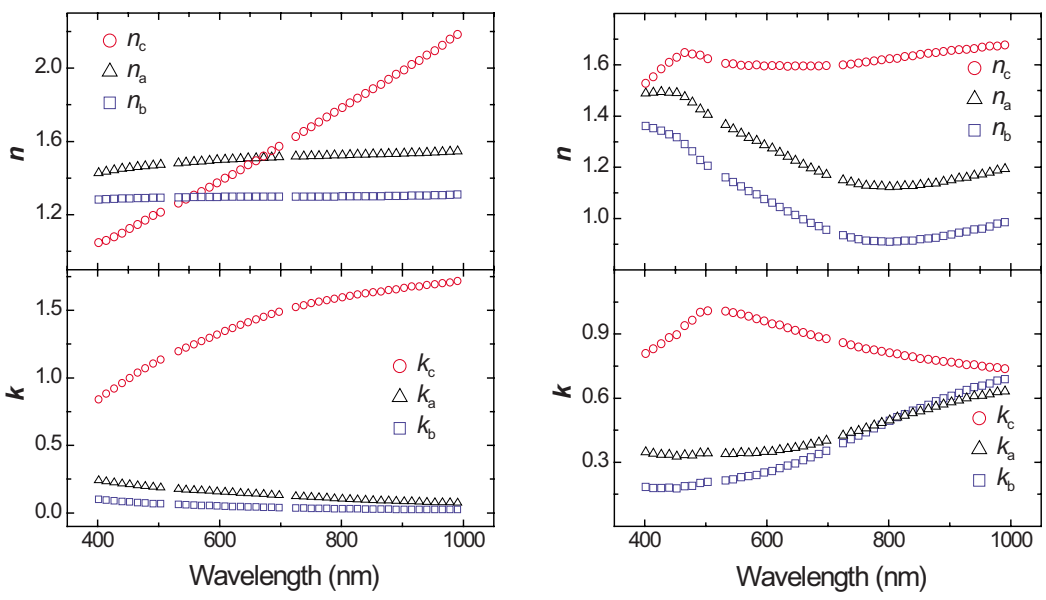

FIG. 5. (Color online) Optical constants of the slanted Co nanocolumns (left) and Co nanocoils (right), respectively. Strong birefringence and dichroism can be observed in the optical constants of both nanostructures. Indices $a, b$, and $c$ correspond to axes shown in Fig. 1. Note that wavelengths around 520 and $720 \mathrm{~nm}$ are disregarded due to detector noise. 


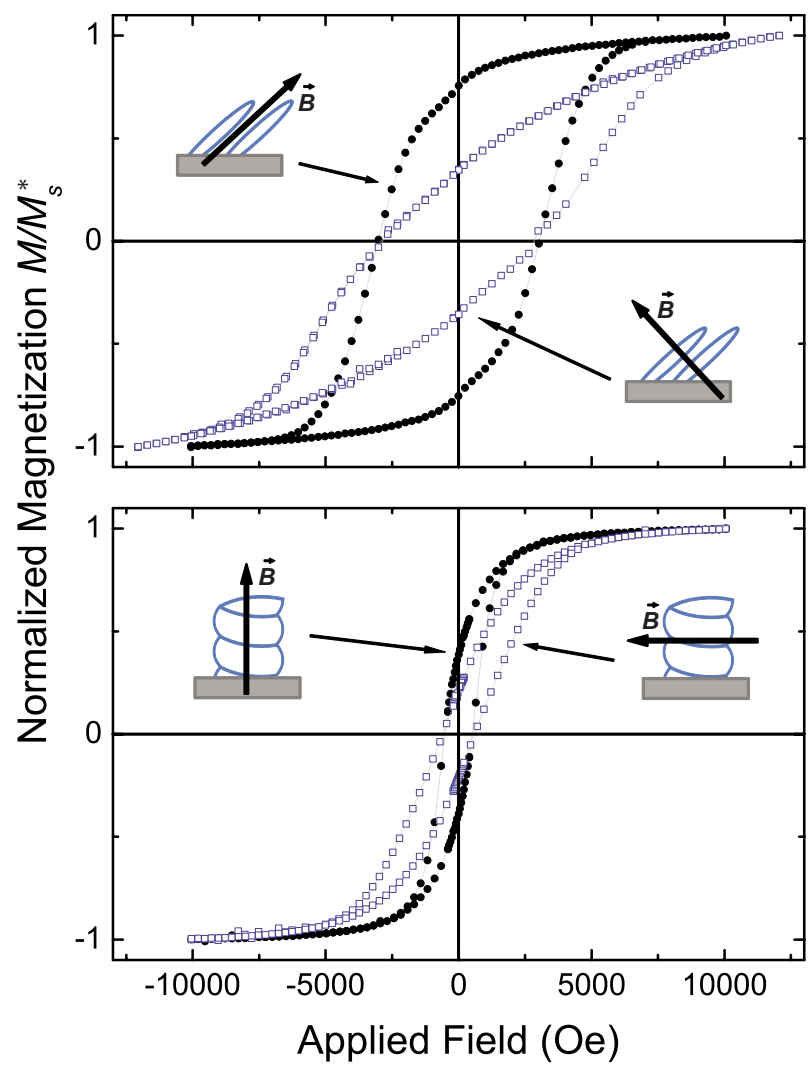

FIG. 6. (Color online) Room temperature magnetic hysteresis loops of slanted Co nanocolumns (top) and Co nanocoils (bottom). The schematics indicate the direction of the magnetic $\mathbf{B}$-field with respect to the nanostructures. A strong coercive field $H_{c} \approx 3 \mathrm{kOe}$ is observed for the slanted nanocolumns.

$H_{c}$ for parallel and perpendicular measurements are very similar but we found large anisotropy with respect to the remanence normalized to $M_{s}^{*}\left(M_{r} / M_{s}^{*}\right)$, which is along the long axis of the structures roughly twice as large as perpendicular to it. This observation is true for both samples under investigation. The extrinsic magnetic properties listed in Table II fall in the range observed in electrodeposited nanowire arrays where the nanowires with similar diameter are perpendicular to the substrate surface. ${ }^{36,37,39}$ Large coercivities of thin wires, for example, are due to magnetic anisotropy, which is largely of the shape-anisotropy type, probably with some random-anisotropy corrections. ${ }^{40,41}$ However, the present geometries, as outlined in Fig. 1, yield a far-reaching modification of the mechanism of magnetization reversal. As we will show elsewhere, this requires the computation and analysis of the demagnetizing-field tensor, which may be interpreted as a magnetostatic analog to Eq. (1) and involves similar angles. The revolution axis of the columns is the

TABLE II. Summary of coercive fields $\left(H_{c}\right)$ and remanent magnetizations normalized to maximum saturation magnetization reached within our experiment $\left(M_{r} / M_{s}^{*}\right)$ of our nanostructured Co thin films.

\begin{tabular}{lcccccc}
\hline \hline & \multicolumn{2}{c}{ Slanted columns } & & \multicolumn{2}{c}{ Nanocoils } \\
\cline { 2 - 3 } \cline { 5 - 6 } & $\|$ & $\perp$ & & $\|$ & $\perp$ \\
\hline$H_{c}$ & $3 \mathrm{kOe}$ & $2.9 \mathrm{kOe}$ & & $0.5 \mathrm{kOe}$ & $0.6 \mathrm{kOe}$ \\
$M_{r} / M_{s}^{*}$ & 0.76 & 0.35 & & 0.39 & 0.21 \\
\hline \hline
\end{tabular}

easier direction of magnetization, which is in agreement with reports of ferromagnetic nanocolumns deposited at an angle of incidence greater than $70^{\circ} .{ }^{9}$ Furthermore, a significant difference in the saturation field parallel and perpendicular to the long axis of the structures, especially for the slanted columnar thin film, hints to a one-dimensional structure. ${ }^{42}$

\section{CONCLUSION}

GE for wavelengths from 400 to $1000 \mathrm{~nm}$ and a SQUID magnetometer were applied to study the optical, structural, and magnetic properties of highly orientationally coherent chiral and achiral slanted cobalt nanostructure thin films. The nanostructure samples deposited by GLAD at $85^{\circ}$ particle flux incidence reveal strongly anisotropic optical properties, as well as strongly anisotropic magnetic properties. Structural analysis indicates growth of hcp structure with $\mathbf{c}$-axis along the nanowire directions. Two different optical models were employed for best-match analysis of the optical spectra for the chiral and achiral samples, induced by substrate rotation and fixture, respectively, and revealed the internal geometrical arrangement of the nanostructures in excellent agreement with SEM images. Strong optical birefringence, dichroism, and dispersion are quantified for both samples, and with sets of optical constants substantially different from bulk Co. The obtained optical constants and shape-induced anisotropy suggest such nanostructures for future tailoring of optical properties for nanostructure thin films. In particular, the achiral slanted nanocolumn thin films are found monoclinic due to dielectric polarization charge coupling effects across neighboring slanted but electrically isolated nanocolumns. Room temperature magnetization hysteresis measurements reveal strong anisotropy with respect to the nanostructure long axes, and a strong coercive field of $3 \mathrm{kOe}$ was measured for the slanted nanostructures. The latter finding is of interest for future use of low-aspect ratio magnetic slanted nanostructures for nanodevice applications such as integrated nanomagnetic memory or nanomagnetic logic applications.

\section{ACKNOWLEDGMENTS}

The authors acknowledge financial support from the National Science Foundation in SGER (Grant No. ECCS0824920), CAREER (Grant No. ECCS-0846329), and MRSEC (Grant No. DMR-0820521), University of NebraskaLincoln, College of Engineering, and J.A. Woollam Foundation.

${ }^{1}$ K. Robbie and M. J. Brett, J. Vac. Sci. Technol. A 15, 1460 (1997).

${ }^{2}$ J. J. Steele, J. P. Gospodyn, J. C. Sit, and M. J. Brett, IEEE Sens. J. 6, 24 (2006).

${ }^{3}$ S. V. Kesapragada, P. Victor, O. Nalamasu, and D. Gall, Nano Lett. 6, 854 (2006).

${ }^{4}$ S. R. Kennedy and M. J. Brett, Appl. Opt. 42, 4573 (2003).

${ }^{5}$ K. Robbie, Y. Cui, C. Elliott, and K. Kaminska, Appl. Opt. 45, 8298 (2006).

${ }^{6}$ I. J. Hodgkinson and Q. H. Wu, Birefringent Thin Films and Polarizing Elements (World Scientific, Singapore, 1998).

${ }^{7}$ A. Lakthakia and R. Messier, Sculptured Thin Films (SPIE, Bellingham, WA, 2004).

${ }^{8}$ G. L. J. A. Rikken and E. Raupach, Nature (London) 390, 493 (1997).

${ }^{9}$ M. M. Hawkeye and M. J. Brett, J. Vac. Sci. Technol. A 25, 1317 (2007).

${ }^{10}$ F. Liu, C. Yu, L. Shen, J. Barnard, and G. J. Mankey, IEEE Trans. Magn. 
36, 2939 (2000).

${ }^{11}$ A. Lisfi and J. C. Lodder, Phys. Rev. B 63, 174441 (2001).

${ }^{12}$ B. Dick, M. J. Brett, T. J. Smy, M. R. Freeman, M. Malac, and R. F. Egerton, J. Vac. Sci. Technol. A 18, 1838 (2000).

${ }^{13}$ F. Tang, D.-L. Liu, D.-X. Ye, Y.-P. Zhao, T.-M. Lu, G.-C. Wang, and A. Vijayaraghavan, J. Appl. Phys. 93, 4194 (2003).

${ }^{14}$ G. W. Mbise, D. L. Bellac, G. A. Niklasson, and C. G. Granqvist, J. Phys. D 30, 2103 (1997).

${ }^{15}$ P. I. Rovira, R. A. Yarussi, R. W. Collins, R. Messier, V. C. Venugopal, A. Lakhtakia, K. Robbie, and M. J. Brett, Appl. Phys. Lett. 71, 1180 (1997).

${ }^{16}$ M. O. Jensen and M. J. Brett, Opt. Express 13, 3348 (2005).

${ }^{17}$ Z.-Y. Zhang and Y.-P. Zhao, J. Appl. Phys. 104, 013517 (2008).

${ }^{18}$ I. S. Nerbø, M. Kildemo, S. L. Roy, I. Simonsen, E. Søndergård, L. Holt, and J. C. Walmsley, Appl. Opt. 47, 5130 (2008).

${ }^{19}$ K. Kaminska, A. Amassian, L. Martinu, and K. Robbie, J. Appl. Phys. 97, 013511 (2005)

${ }^{20}$ S.-H. Hsu, E.-S. Liu, Y.-C. Chang, J. N. Hilfiker, Y. D. Kim, T. J. Kim, C.-J. Lin, and G.-R. Lin, Phys. Status Solidi A 205, 876 (2008).

${ }^{21}$ G. Beydaghyan, C. Buzea, Y. Chi, C. Elliott, and K. Robbie, Appl. Phys. Lett. 87, 153103 (2005).

${ }^{22}$ D. E. Aspnes, Thin Solid Films 89, 249 (1982).

${ }^{23}$ M. Schubert and W. Dollase, Opt. Lett. 27, 2073 (2002).

${ }^{24}$ M. Dressel, B. Gompf, D. Faltermeier, A. K. Tripathi, J. Pflaum, and M. Schubert, Opt. Express 16, 19770 (2008).

${ }^{25}$ D. Schmidt, B. Booso, T. Hofmann, E. Schubert, A. Sarangan, and M. Schubert, Appl. Phys. Lett. 94, 011914 (2009).

${ }^{26}$ D. Schmidt, B. Booso, T. Hofmann, E. Schubert, A. Sarangan, and M.
Schubert, Opt. Lett. 34, 992 (2009).

${ }^{27} \mathrm{M}$. Schubert, Introduction to Complex Mediums for Optics and Electromagnetics (SPIE, Bellingham, WA, 2003), pp. 677-706.

${ }^{28}$ M. Schubert, Ann. Phys. 15, 480 (2006).

${ }^{29}$ Handbook of Ellipsometry, edited by H. G. Tompkins and E. A. Irene (Springer, Heidelberg, 2004).

${ }^{30}$ M. Schubert, Phys. Rev. B 53, 4265 (1996).

${ }^{31}$ M. Schubert, Thin Solid Films 313-314, 323 (1998).

${ }^{32}$ M. Schubert, Infrared Ellipsometry on Semiconductor Layer Structures (Springer, Heidelberg, 2004).

${ }^{33}$ D. Schmidt, E. Schubert, and M. Schubert, Phys. Status Solidi A 205, 748 (2008).

${ }^{34}$ I.-H. Suh, Y.-S. Park, and J.-G. Kim, J. Appl. Crystallogr. 33, 994 (2000). ${ }^{35}$ M. Schubert and C. M. Herzinger, Phys. Status Solidi A 188, 1563 (2001).

${ }^{36}$ Y. Henry, K. Ounadjela, L. Piraux, S. Dubois, J.-M. George, and J.-L. Duvail, Eur. Phys. J. B 20, 35 (2001).

${ }^{37}$ M. Darques, L. Piraux, A. Encinas, P. Bayle-Guillemaud, A. Popa, and U. Ebels, Appl. Phys. Lett. 86, 072508 (2005).

${ }^{38}$ Handbook of Optical Constants of Solids, edited by E. D. Palik (Academic, Boston, MA, 1991).

${ }^{39}$ D. J. Sellmyer, M. Zheng, and R. Skomski, J. Phys.: Condens. Matter 13, R433 (2001).

${ }^{40}$ R. Skomski, H. Zeng, M. Zheng, and D. J. Sellmyer, Phys. Rev. B 62, 3900 (2000).

${ }^{41}$ R. Skomski, J. Phys.: Condens. Matter 15, R841 (2003).

${ }^{42}$ T. M. Whitney, J. S. Jiang, P. C. Searson, and C. L. Chien, Science 261, 1316 (1993). 